Marek Golasiński@

\title{
Homotopy nilpotency of some homogeneous spaces
}

Received: 6 July 2020 / Accepted: 6 January 2021 / Published online: 4 February 2021

\begin{abstract}
Let $\mathbb{K}=\mathbb{R}, \mathbb{C}$, the field of reals or complex numbers and $\mathbb{H}$, the skew $\mathbb{R}$ algebra of quaternions. We study the homotopy nilpotency of the loop spaces $\Omega\left(G_{n, m}(\mathbb{K})\right)$, $\Omega\left(F_{n ; n_{1}, \ldots, n_{k}}(\mathbb{K})\right)$, and $\Omega\left(V_{n, m}(\mathbb{K})\right)$ of Grassmann $G_{n, m}(\mathbb{K})$, flag $F_{n ; n_{1}, \ldots, n_{k}}(\mathbb{K})$ and Stiefel $V_{n, m}(\mathbb{K})$ manifolds. Additionally, homotopy nilpotency classes of $p$-localized $\Omega\left(G_{n, m}^{+}(\mathbb{K})_{(p)}\right)$ and $\Omega\left(V_{n, m}(\mathbb{K})_{(p)}\right)$ for certain primes $p$ are estimated, where $G_{n, m}^{+}(\mathbb{K})_{(p)}$ is the oriented Grassmann manifolds. Further, the homotopy nilpotency classes of loop spaces of localized homogeneous spaces given as quotients of exceptional Lie groups are investigated as well.
\end{abstract}

\section{Introduction}

The homotopy nilpotency classes nil $X$ of associative $H$-spaces $X$ has been extensively studied in addition to their homotopy commutativity. In particular, Hopkins [8] made great progress by giving (co)homological criteria for homotopy associative finite $H$-spaces to be homotopy nilpotent. For example, he showed that if a homotopy associative finite $H$-space has no torsion in the integral homology, then it is homotopy nilpotent. Later, Rao $[19,20]$ showed that the converse of the above criterion is true in the case of groups $\operatorname{Spin}(n)$ and $S O(n)$ and a connected compact Lie group is homotopy nilpotent if and only if it has no torsion in homology. Eventually, Yagita [26] proved that, when $G$ is a compact, simply connected Lie group, its $p$-localization $G_{(p)}$ is homotopy nilpotent if and only if it has no torsion in the integral homology. Although many results on the homotopy nilpotency are known, precise homotopy nilpotence classes have not been determined in most cases.

Let $\mathbb{K} P^{n}$ be the projective $n$-space for $\mathbb{K}=\mathbb{R}, \mathbb{C}$, the field of reals or complex numbers and $\mathbb{H}$, the skew $\mathbb{R}$-algebra of quaternions. The homotopy nilpotency of the loop spaces $\Omega\left(\mathbb{K} P^{n}\right)$ has been first studied by Ganea [5], Snaith [22] and then their $p$-localization $\Omega\left(\left(\mathbb{K} P^{n}\right)_{(p)}\right)$ by Meier [15].

For the James reduced product $J(X)$ of a space $X$, Cohen and $\mathrm{Wu}$ [4] have asked:

QUESTION. Is the Cohen group $[J(X), \Omega(Y)]$ nilpotent for any spaces $X, Y$ ?

Marek Golasiński (凶): Faculty of Mathematics and Computer Science, University of Warmia and Mazury, Słoneczna 54 Street, 10-710 Olsztyn, Polande-mail: marekg@matman.uwm.edu.pl

Mathematics Subject Classification (2020) Primary: 55P15; Secondary: 55P35 - 55T20 
We were first inspired by the homotopy nilpotency of $\Omega\left(\mathbb{H} P^{n}\right)$ for any $n \geq 1$ which does not appear in the literature known to the author and then by the question above as well. The paper grew out of our desire to develop techniques in calculating homotopy nilpotency classes of loop spaces of homogeneous spaces. Because no answer is known to the question above, we present some homogeneous spaces $Y$ such that the group $[J(X), \Omega(Y)]$ is nilpotent for any space $X$.

In Section 1, we set stages for developments to come. This introductory section is devoted to a general discussion and establishes notations on homotopy nilpotency of associative $H$-spaces used in the rest of the paper.

Section 2, based on results [27, Chapter II] by Zabrodsky, takes up the systematic study of the homotopy nilpotency of loop spaces $\Omega\left(G_{n, m}(\mathbb{K})\right)$ and $\Omega\left(V_{n, m}(\mathbb{K})\right)$ of Grassmann $G_{n, m}(\mathbb{K})$ and Stiefel $V_{n, m}(\mathbb{K})$ manifolds. First, we make use of [27, Lemma 2.6.6] to derive:

Propoition 2.2. If $G$ is a compact Lie group and $K<G$ its closed subgroup with nil $K<\infty$ then nil $\Omega(G / K)<\infty$.

Then, we obtain the following results:

Propoition 2.7. If $1 \leq m \leq n \leq \infty$ then:

(1) $\operatorname{nil} \Omega\left(G_{n, m}^{+}(\mathbb{R})_{(p)}\right)<\infty$ for $p \geq 3$;

(2) $\operatorname{nil} \Omega\left(G_{n, m}(\mathbb{K})\right)<\infty$ for $\mathbb{K}=\mathbb{C}, \mathbb{H}$.

In particular, nil $\Omega\left(\mathbb{H} P^{n}\right)<\infty$.

Certainly, for (oriented) flag $F_{n ; n_{1}, \ldots, n_{k}}(\mathbb{K})$ manifolds, Proposition 2.2 leads to:

(1) $\operatorname{nil} \Omega\left(F_{n ; n_{1}, \ldots, n_{k}}^{+}(\mathbb{R})_{(p)}\right)<\infty$ and nil $\Omega\left(F_{n ; n_{1}, \ldots, n_{k}}(\mathbb{R})_{(p)}\right)<\infty$ for $p \geq 3$;

(2) $\operatorname{nil} \Omega\left(F_{n ; n_{1}, \ldots, n_{k}}(\mathbb{K})\right)<\infty$ for $\mathbb{K}=\mathbb{C}, \mathbb{H}$.

Proposition 2.17. If $1 \leq m \leq n$ then:

(1) $\operatorname{nil} \Omega\left(\left(V_{n, m}\right)_{(p)}\right)<\infty$ for $p \geq 3$;

(2) $\operatorname{nil} \Omega\left(W_{n, m}\right)<\infty$;

(3) $\operatorname{nil} \Omega\left(X_{n, m}\right)<\infty$.

Corollaries 2.4, 2.9 and 2.18 answer Cohen-Wu question [4] for some homogeneous spaces given as quotients of classical Lie groups. In particular, for Grassmann, flag and Stiefel manifolds.

Next, we make use of results [10,11] by Kaji and Kishimoto, [12] by Kishimoto and [14] by McGibbon on $p$-regular classical groups to estimate the homotopy nilpotency classes of the $p$-localized spaces $\Omega\left(G_{n, m}(\mathbb{K})_{(p)}\right)$ and $\Omega\left(V_{n, m}(\mathbb{K})_{(p)}\right)$ for $\mathbb{K}=\mathbb{R}, \mathbb{C}$, the field of reals or complex numbers and $\mathbb{H}$, the skew $\mathbb{R}$-algebra of quaterions.

Section 3 is devoted to the homotopy nilpotency of the loop spaces $\Omega\left(\mathbb{O} P^{2}\right)$ of the Cayley plane $\mathbb{O} P^{2}$ and of other homogeneous spaces being quotients of exceptional Lie groups. We also make use results [24, Theorem 12.1] by Theriault on the homotopy nilpotency classes of quasi- $p$-regular exceptional Lie groups to estimate the homotopy nilpotency classes of $p$-localized loop spaces of those homogeneeous spaces. 


\section{Preliminaries}

All spaces and maps in this note are assumed to be connected, based and of the homotopy type of $C W$-complexes. We also do not distinguish notationally between a continuous map and its homotopy class. We write $\Omega(X)(\operatorname{resp} . \Sigma(X))$ for the loop (resp. suspension) space on a space $X$ and $[Y, X]$ for the set of homopoty classes of maps $Y \rightarrow X$.

Given a space $X$, we use the customary notations $X \vee X$ and $X \wedge X$ for the wedge and the smash product of $X$, respectively.

Recall that an $H$-space is a pair $(X, \mu)$, where $X$ is a space and $\mu: X \times X \rightarrow X$ is a map such that the diagram

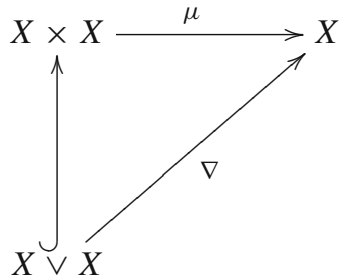

commutes up to homotopy, where $\nabla: X \vee X \rightarrow X$ is the codiagonal map.

We call $\mu$ a multiplication or an $H$-structure for $X$. Two examples of $H$-spaces come in mind: topological groups and the spaces $\Omega(X)$ of loops on $X$. In the sequel, we identify an $H$-space $(X, \mu)$ with the space $X$.

An $H$-space $X$ is called a group-like space if $X$ satisfies all the axioms of groups up to homotopy. Recall that a homotopy associative an $H$-space always has a homotopy inverse. More precisely, according to [27, 1.3.2. Corollary] (see also [1, Proposition 8.4.4]), we have:

Proposition 1.1. If $X$ is a homotopy associative $H$-space then $X$ is a group-like space.

If $X$ is a homotopy associative $H$-space, then the functor $[-, X]$ takes its values in the category of groups. One may then ask when this functor takes its values in various subcategories of groups.

For example, $X$ is homotopy commutative if and only if $[Y, X]$ is abelian for all $Y$.

Given a group-like space $X$, we write $\gamma_{X, 1}=\operatorname{id}_{X}: X \rightarrow X$ and $\bar{\gamma}_{X, 2}:$ $X \times X \rightarrow X$ for the commutator map of $X$. Observe that the restriction of $\bar{\gamma}_{X, 2}$ to the wedge $X \vee X$ is null homotopic so $\bar{\gamma}_{X, 2}$ extends to a map

$$
\gamma_{X, 2}: X \wedge X \rightarrow X
$$

For an integer $n \geq 1$, let $X^{\wedge(n+1)}$ be the $(n+1)$-fold smash power of $X$. Define the iterated Samelson product

$$
\gamma_{X, n+1}: X^{\wedge(n+1)} \rightarrow X
$$

inductively by $\gamma_{X, n+1}=\gamma_{X, 2} \circ\left(\gamma_{X, 1} \wedge \gamma_{X, n}\right)$ for $n \geq 2$. Notice that $\gamma_{X, n}$ has a universal property: any Samelson product of length $n$ on $X$ factors through $\gamma_{X, n}$. 
Lemma 1.2. If $X$ is a homotopy associative $H$-space and $Y$ a finite dimensional $C W$-complex with $\operatorname{dim} Y=n$ then the group $[Y, X]$ is nilpotent with the nilpotency class at most $n$.

Proof. First, recall that given a homotopy associative $H$-space $X$, in view of [9], all its $m$-th Postnikov stages $P_{m} X$ are also a homotopy associative $H$-space and the canonical map $X \rightarrow P_{m} X$ is an $H$-map. Hence, for a $C W$-complex $Y$ with $\operatorname{dim} Y=$ $n$, there is an isomorphism $[Y, X] \approx\left[Y, P_{n} X\right]$ determined by the canonical map $X \rightarrow P_{n} X$. Then, the map $\gamma_{P_{n} X, n+1}\left(f_{1} \wedge \cdots \wedge f_{n+1}\right): Y^{\wedge(n+1)} \rightarrow P_{n} X$ is homotopy trivial for any maps $f_{1}, \ldots, f_{n+1}: Y \rightarrow P_{n} X$ since the space $Y^{\wedge(n+1)}$ is $n$-connected. Consequently, nil $[Y, X]=\operatorname{nil}\left[Y, P_{n} X\right] \leq n$ and the proof follows.

We point out that the result above has been stated in $[8,20]$ but for a finite $C W$-complex and a finite homotopy associative $H$-space $X$, only. Next, any $C W$ complex $Y$ can be expressed as

$$
Y=\lim _{\rightarrow} Y_{\alpha}
$$

with $Y_{\alpha}$ finite. This leads to the short exact sequence

$$
1 \rightarrow \lim _{\leftarrow}^{1}\left[\Sigma Y_{\alpha}, X\right] \longrightarrow[Y, X] \longrightarrow \lim _{\leftarrow}\left[Y_{\alpha}, X\right] \rightarrow 1
$$

for any connected homotopy associative $H$-space $X$.

In view of [8, Proposition 1.2], we have that the intersection $\Gamma_{d} \cap\left(\lim _{\leftarrow}^{1}\left[\Sigma Y_{\alpha}, X\right]\right)$ $=0$ for any finite connected, homotopy associative $H$-space $X$ with $\operatorname{dim} X=d<$ $\infty$, where $\Gamma_{d}$ stands for the $d$-th member of the lower central series of the group $[Y, X]$. Then, Lemma 1.2 and [8, Proposition 1.1] yield:

Proposition 1.3. If $X$ is a finite connected, homotopy associative $H$-space then the group $[Y, X]$ is pro-nilpotent for any $C W$-complex $Y$.

One might ask if there is an upper bound for the nilpotency class of $[Y, X]$ that is independent of $Y$. The homotopy nilpotency class of $X$ is the least $n$ such that $\gamma_{X, n+1} \simeq *$ and $\gamma_{X, n} \not \approx *$. In this case, we write nil $X=n$ and call the homotopy associative $H$-space $X$ homotopy nilpotent.

Note that nil $X=1$ if and only if $X$ is homotopy commutative.

By [2, Theorem 2.7], one has:

$$
\operatorname{nil} X=\sup _{m} \operatorname{nil}\left[X^{m}, X\right]=\sup _{Y} \operatorname{nil}[Y, X]
$$

for the $m$-th Cartesian power $X^{m}$ of $X$.

Given a space $X$, the number nil $\Omega(X)$ (if any) is called the homotopy nilpotency class of $X$.

Further, according to Zabrodsky [27, Lemma 2.6.1], we have:

Proposition 1.4. A connected associative $H$-space $X$ is homotopy nilpotent if and only if the functor $[-, X]$ on the category of all pointed spaces is nilpotent group valued. 
It is obvious that for homotopy nilpotent group-like spaces $X_{1}, \ldots, X_{m}$, we have

$$
\operatorname{nil}\left(X_{1} \times \cdots \times X_{m}\right)=\max \left\{\operatorname{nil} X_{1}, \ldots, \operatorname{nil} X_{m}\right\}
$$

The first major advance was made by Hopkins [8, Theorem 2.1] (completed by Rao [20, Theorem 0.2]). He showed that a finite $H$-space $X$ is homotopy nilpotent if and only if for sufficiently large $n, \gamma_{X, n}$ 's induce trivial homomorphism in complex bordism. This is same as asking that $\gamma_{X, n}$ 's induce trivial homomorphisms in all Morava $K$-theories.

Then, in [8, Corollary 2.2], it was deduced:

Corollary 1.5. If $X$ is a finite associative $H$-space and the homology $H_{*}(X, \mathbb{Z})$ is torsion free then $X$ is homotopy nilpotent.

This corollary implies:

$$
\text { nil } U(n)<\infty \text { and nil } \operatorname{Sp}(n)<\infty \text {. }
$$

But, the homotopy nilpotency does not imply the nilpotency of a topological group.

Remark 1.6. Since the commutators [SO(3), $S O(3)]=S O(3)$ and $[S U(2)$, $S U(2)]=S U(2)$ and: $S O(3) \subseteq S O(n) \subseteq O(n)$ for $n \geq 3, S U(2) \subseteq S U(n) \subseteq$ $U(n)$ for $n \geq 2$ and $S U(2)=S p(1) \subseteq S p(n)$ for $n \geq 1$, we derive that the groups:

(1) $S O(n)$ and $O(n)$ are not nilpotent for $n \geq 3$;

(2) $U(n)$ and $S U(n)$ are not nilpotent for $n \geq 2$;

(3) $\operatorname{Sp}(n)$ is not nilpotent for $n \geq 1$.

Later, Rao [19] showed that the converse of the criterion from Corollary 1.5 is true in the case of $\operatorname{Spin}(n)$ and $S O(n)$ by showing that $\operatorname{Spin}(n), S O(n), n \geq 7$ and $S O(3), S O(4)$ are not homotopy nilpotent.

Because of $H$-homotopy equivalences $O(n) \simeq S O(n) \times \mathbb{Z}_{2}$ and $U(n) \simeq$ $S U(n) \times \mathbb{S}^{1}$, we derive:

$$
\operatorname{nil} S O(n)=\operatorname{nil} O(n) \text { and } \operatorname{nil} S U(n)=\operatorname{nil} U(n)<\infty .
$$

Next, write $O=\lim _{\rightarrow} O(n), U=\lim _{\rightarrow} U(n)$ and $S p=\lim _{\rightarrow} S p(n)$. Then, notice that by Bott periodicity theorem: $\Omega^{8}(O) \simeq O, \Omega^{2}(U) \simeq U$ and $\Omega^{8}(S p) \simeq S p$, we get

$$
\operatorname{nil} O=\operatorname{nil} U=\operatorname{nil} S p=1 .
$$

Let now $G_{(p)}$ stand for the $p$-localization in the sense of [3] of a compact Lie group $G$. Then, Yagita [26, Theorem] has shown:

Theorem 1.7. Let $G$ be a simply connected Lie group. Then for each prime $p$, the p-localization $G_{(p)}$ is homotopy nilpotent if and only if the integral cohomology $H^{*}(G, \mathbb{Z})$ has no p-torsion.

Next, Rao [20, Theorem 0.2] has generalized Theorem 1.7 as follows: 
Theorem 1.8. Let $G$ be a compact connected Lie group and let $p$ be a prime. Then $G_{(p)}$ is homotopy nilpotent if and only if $H_{*}\left(G, \mathbb{Z}_{(p)}\right)$ is torsion-free.

Now, write $J(X)$ for the James reduced product on a space $X$ and $B G$ for the classifying space of a topological group $G$. In view of [23, Theorem 8.6], there is an $H$-map $G \rightarrow \Omega(B G)$ which is a weak homotopy equivalence. Consequently, for a Lie group $G$ there is an $H$-homotopy equivalence $\Omega(B G) \simeq G$. Then, for the question [4] by Cohen and $\mathrm{Wu}$, we conclude:

Corollary 1.9. If $n \geq 1$ and $X$ is a space then:

(1) nil $\left[J(X), \Omega\left(B O(n)_{(p)}\right)\right]=\operatorname{nil}\left[J(X), \Omega\left(B S O(n)_{(p)}\right)\right]<\infty$ for any prime $p \geq 3$

(2) nil $[J(X), \Omega(B U(n))]=\operatorname{nil}[J(X), \Omega(B S U(n))]<\infty$;

(3) $\operatorname{nil}[J(X), \Omega(B S p(n))]<\infty$.

\section{Main results}

Let $f: X \rightarrow Y$ be an $H$-map of homotopy associative $H$-spaces. Recall from [27, Chapter II] that:

(1) it is said nil $f \leq n$ if $f \gamma_{X, n} \simeq *$;

(2) $f$ is called central if $\gamma_{Y, 2}\left(f \wedge \mathrm{id}_{Y}\right) \simeq *$.

Notice that nil $f \leq \min \{\operatorname{nil} X$, nil $Y\}$.

Then, in view of [27, Lemma 2.6.6], we have:

Lemma 2.1. Let $F \stackrel{i}{\rightarrow} E \stackrel{q}{\rightarrow} B$ be an $H$-fibration, i.e., $F \stackrel{i}{\rightarrow} E \stackrel{q}{\rightarrow} B$ is a fibration, $F, E$ and $B$ are $H$-spaces and the maps $i: F \rightarrow E$, and $q: E \rightarrow B$ are $H$-maps.

(1) If nil $q \leq n$ and $i: F \rightarrow E$ is central then nil $E \leq n+1$;

(2) if $\Omega(Y) \stackrel{i}{\rightarrow} E \stackrel{q}{\rightarrow} X$ is the induced $H$-fibration by an $H$-map $f: X \rightarrow Y$ then the map $i: \Omega(Y) \rightarrow E$ is central.

If a topological group $G$ acts freely on a paracompact spaces $X$ then there is a homeomorphism

$$
X / G \approx X \times_{G} E G,
$$

where $G \rightarrow E G \rightarrow B G$ is the universal principal $G$-bundle.

Since the connecting map $\partial_{X}: \Omega(X / G) \rightarrow G$, in view of [23, Theorem 8.6] (see also [6, Corollary 3.4]), is an $H$-map, the fibration $X \rightarrow X / G \rightarrow E G / G \approx$ $B G$ leads to the $H$-fibration

$$
\Omega(X) \longrightarrow \Omega(X / G) \stackrel{\partial_{X}}{\longrightarrow} G
$$

Now, let $G$ be a compact Lie group and $K<G$ its closed subgroup. Then, the quotient space $G / K$ is a manifold and the quotient map $q: G \rightarrow G / K$ is a submersion. Hence, $q: G \rightarrow G / K$ has a local section at the point $q(e)=K$ for the unit $e \in G$. This certainly implies that the $\operatorname{map} q: G \rightarrow G / K$ has a local section at 
any point $q(g)$ for any $g \in G$. Consequently, the quotient map $q: G \rightarrow G / K$ is a fiber bundle with the fiber $K$ as a principal $K$-bundle. Thus, we have a $K$-fibration

$$
\Omega(G) \longrightarrow \Omega(G / K) \stackrel{\partial_{G}}{\longrightarrow} K .
$$

Since this fibration is induced by the inclusion map $K \hookrightarrow G$ which is certainly a $K$-map, Lemma 2.1 yields:

Proposition 2.2. If $G$ is a compact Lie group and $K<G$ its closed subgroup with nil $K<\infty$ then nil $\Omega(G / K)<\infty$.

Because of the inclusion maps $U(n) \hookrightarrow S O(2 n), U(n) \hookrightarrow S p(n)$ and $S p(n) \hookrightarrow S U(2 n) \hookrightarrow S O(4 n)$, we get the $H$-fibrations:

(1) $\Omega(S O(2 n)) \rightarrow \Omega(S O(2 n) / U(n)) \rightarrow U(n)$;

(2) $\Omega(S p(n)) \rightarrow \Omega(S p(n) / U(n)) \rightarrow U(n)$;

(3) $\Omega(S U(2 n)) \rightarrow \Omega(S U(2 n) / S p(n)) \rightarrow S p(n)$;

(4) $\Omega(S O(4 n)) \rightarrow \Omega(S O(4 n) / S p(n)) \rightarrow S p(n)$.

Then, the fibrations above, Corollary 1.5 and Proposition 2.2 lead to:

Proposition 2.3. If $n \geq 1$ then:

(1) $\operatorname{nil} \Omega(S O(2 n) / U(n))<\infty$;

(2) $\operatorname{nil} \Omega(S p(n) / U(n))<\infty$;

(3) $\operatorname{nil} \Omega(S U(2 n) / S p(n))<\infty$;

(4) $\operatorname{nil} \Omega(S O(4 n) / S p(n))<\infty$.

For the question [4] by Cohen and $\mathrm{Wu}$, we conclude:

Corollary 2.4. If $n \geq 1$ then:

(1) $\operatorname{nil}[J(X), \Omega(S O(2 n) / U(n))]<\infty$;

(2) $\operatorname{nil}[J(X), \Omega(S p(n) / U(n))]<\infty$;

(3) $\operatorname{nil}[J(X), \Omega(S U(2 n) / S p(n))]<\infty$;

(4) $[J(X), \Omega(S O(4 n) / S p(n))]<\infty$

for any space $X$.

\subsection{Grassmannians}

Let $\mathbb{K}=\mathbb{R}$, $\mathbb{C}$ be the field of reals or complex numbers and $\mathbb{H}$, the skew $\mathbb{R}$-algebra of quaternions. Then, we set:

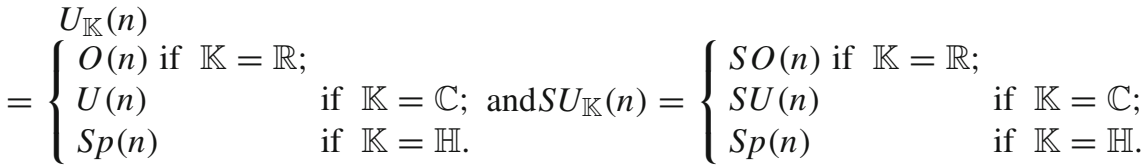

Write $G_{n, m}(\mathbb{K})$ (resp. $G_{n, m}^{+}(\mathbb{K})$ ) for the (resp. oriented) Grassmannian of $m$ dimensional subspaces in the $n$-dimensional $\mathbb{K}$-vector space. For example, the set of lines $G_{n+1,1}(\mathbb{K})=\mathbb{K} P^{n}$, the projective $n$-space over $\mathbb{K}$. 
It is well known that $G_{n, m}(\mathbb{K})$ (resp. $\left.G_{n, m}^{+}(\mathbb{K})\right)$ are smooth manifolds with diffeomorphisms

$$
\begin{aligned}
& G_{n, m}(\mathbb{K}) \approx\left(U_{\mathbb{K}}(n) / U_{\mathbb{K}}(m) \times U_{\mathbb{K}}(n-m)\right) \text { and } G_{n, m}^{+}(\mathbb{K}) \\
& \quad \approx\left(S U_{\mathbb{K}}(n) / S U_{\mathbb{K}}(m) \times S U_{\mathbb{K}}(n-m)\right)
\end{aligned}
$$

for $\mathbb{K}=\mathbb{R}, \mathbb{C}, \mathbb{H}$.

Since the homomorphism $\pi_{1}\left(S U(m)_{\mathbb{K}}\right) \rightarrow \pi_{1}\left(S U(n)_{\mathbb{K}}\right)$ of fundamental groups determined by the inclusion map $S U(m)_{\mathbb{K}} \hookrightarrow S U(n)_{\mathbb{K}}$ for $2 \leq m \leq n$ is an epimorphism, we derive that the spaces $G_{n, m}^{+}(\mathbb{K})$ are simply connected for $\mathbb{K}=\mathbb{R}, \mathbb{C}, \mathbb{H}$. Next, there is the universal covering map

$$
\mathbb{Z}_{2} \longrightarrow G_{n, m}^{+}(\mathbb{R}) \longrightarrow G_{n, m}(\mathbb{R})
$$

and a fibre bundle

$$
\mathbb{S}^{1} \longrightarrow G_{n, m}^{+}(\mathbb{C}) \longrightarrow G_{n, m}(\mathbb{C}) .
$$

Since $G_{n+1,1}(\mathbb{K})=\mathbb{K} P^{n}$, projective spaces are special cases of Grassmannians. Recall that by Ganea [5, Propositions 1.3-1.5] and Snaith [22, Corollary 3.3], we have:

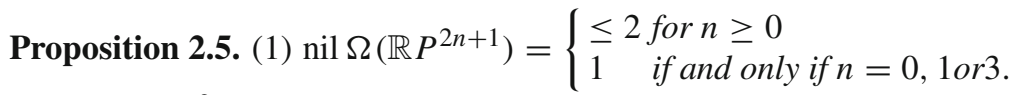

(2) $\operatorname{nil} \Omega\left(\mathbb{R} P^{2 n}\right)=\infty$ for $n \geq 1$.

(3) nil $\Omega\left(\mathbb{C} P^{2 n+1}\right)= \begin{cases}\leq 2 & \text { for any odd } n \geq 1 \\ 1 & \text { if and only if } n=1 .\end{cases}$

(4) $3 \leq$ nil $\Omega\left(\mathbb{C} P^{2 n}\right) \leq 6$ for $n \geq 1$.

(5) $3 \leq \operatorname{nil} \Omega\left(\mathbb{H} P^{n}\right)$ for any $n \geq 1$.

(6) $\operatorname{nil} \Omega\left(\mathbb{H} P^{n}\right)=3$ if $n \equiv-1(\bmod 24)$.

Then, Meier [15, Theorem 5.4] has shown some results on the homotopy nilpotency of $p$-localized projective spaces:

Theorem 2.6. Let $p$ be an odd prime and $n \geq 2$ a natural number. Then:

(1) $\operatorname{nil} \Omega\left(\mathbb{C} P_{(p)}^{n}\right)=1$.

(2) nil $\Omega\left(\mathbb{H} P_{(p)}^{n}\right)=1$ if $p>3$.

(3) $3 \leq \operatorname{nil} \Omega\left(\mathbb{H} P_{(3)}^{n}\right) \leq 4$.

(4) $\operatorname{nil} \Omega\left(\mathbb{H} P_{(3)}^{n}\right)=3$ if $n \equiv 2(\bmod 3)$.

Since the space $\mathbb{R} P^{2 n+1}$ is simple, there is its localization $\mathbb{R} P_{(p)}^{2 n+1}$ for any prime $p \geq 2$. It it also easily to see that nil $\Omega\left(\mathbb{R} P_{(p)}^{2 n+1}\right)=1$ for any odd prime $p$ and $n \geq 0$. But, the nilpotency nil $\Omega\left(\mathbb{H} P^{n}\right)$ for any $n \geq 2$ does not appear in the literature known to the author. Next, recall that the classifying

$$
B U_{\mathbb{K}}(m)=\lim _{\rightarrow} G_{n, m}(\mathbb{K})=G_{\infty, m}(\mathbb{K}) .
$$

Since the cohomology $H^{*}(S O(n), \mathbb{Z})$ has only 2-torsions and $H^{*}(U(n), \mathbb{Z})$, $H^{*}(\operatorname{Sp}(n), \mathbb{Z})$ are torsion free, the fibration

$$
\Omega\left(S U_{\mathbb{K}}(n)\right) \longrightarrow \Omega\left(G_{n, m}^{+}(\mathbb{K})\right) \longrightarrow S U_{\mathbb{K}}(m) \times S U_{\mathbb{K}}(n-m),
$$

Corollary 1.5, Theorem 1.8 and Proposition 2.2 lead to: 
Proposition 2.7. If $1 \leq m<n \leq \infty$ then:

(1) nil $\Omega\left(G_{n, m}^{+}(\mathbb{R})_{(p)}\right)<\infty$ for $p \geq 3$;

(2) nil $\Omega\left(G_{n, m}(\mathbb{K})\right)<\infty$ and nil $\Omega\left(G_{n, m}^{+}(\mathbb{K})\right)<\infty$ for $\mathbb{K}=\mathbb{C}, \mathbb{H}$. In particular, nil $\Omega\left(\mathbb{H} P^{n}\right)<\infty$.

We do not mention above any result on the $p$-localization of $G_{n, m}(\mathbb{R})$ because we are not sure on its existence.

Remark 2.8. The (resp. oriented) flag manifold $F_{n ; n_{1}, \ldots, n_{k}}(\mathbb{K})\left(\operatorname{resp} . F_{n ; n_{1}, \ldots, n_{k}}^{+}(\mathbb{K})\right)$ with $1 \leq n_{1}<\cdots<n_{k} \leq n-1$ in the $n$-dimensional $\mathbb{K}$-vector space is smooth with a diffeomorphism

$F_{n ; n_{1}, \ldots, n_{k}}(\mathbb{K}) \approx\left(U_{\mathbb{K}}(n) / U_{\mathbb{K}}\left(n_{1}\right) \times U_{\mathbb{K}}\left(n_{1}-n_{2}\right) \times \cdots \times U_{\mathbb{K}}\left(n_{k-1}-n_{k}\right) \times U_{\mathbb{K}}(n-\right.$ $\left.\left.n_{k}\right)\right)$ and $F_{n ; n_{1}, \ldots, n_{k}}^{+}(\mathbb{K}) \approx\left(S U_{\mathbb{K}}(n) / S U_{\mathbb{K}}\left(n_{1}\right) \times S U_{\mathbb{K}}\left(n_{1}-n_{2}\right) \times \cdots \times S U_{\mathbb{K}}\left(n_{k-1}-\right.\right.$ $\left.\left.n_{k}\right) \times S U_{\mathbb{K}}\left(n-n_{k}\right)\right)$. Further, $F_{n ; n_{1}, \ldots, n_{k}}(\mathbb{K}) \approx F_{n ; n_{1}, \ldots, n_{k}}^{+}(\mathbb{K})$ for $\mathbb{K}=\mathbb{C}, \mathbb{H}$.

Since the homomorphism $\pi_{1}\left(S U(m)_{\mathbb{K}}\right) \rightarrow \pi_{1}\left(S U(n)_{\mathbb{K}}\right)$ determined by the inclusion map $S U(m)_{\mathbb{K}} \hookrightarrow S U(n)_{\mathbb{K}}$ for $2 \leq m \leq n$ of fundamental groups is an epimorphism, we derive that the spaces $F_{n ; n_{1}, \ldots, n_{k}}^{+}(\mathbb{K})$ are simply connected for $\mathbb{K}=\mathbb{R}, \mathbb{C}, \mathbb{H}$.

Furthermore, there is the universal covering map

$$
\left(\mathbb{Z}_{2}\right)^{k} \rightarrow F_{n ; n_{1}, \ldots, n_{k}}^{+}(\mathbb{R}) \rightarrow F_{n ; n_{1}, \ldots, n_{k}}(\mathbb{R})
$$

and a fibre bundle

$$
\left(\mathbb{S}^{1}\right)^{k} \rightarrow F_{n ; n_{1}, \ldots, n_{k}}^{+}(\mathbb{C}) \rightarrow F_{n ; n_{1}, \ldots, n_{k}}(\mathbb{C})
$$

Then, in view of the fibration

$\Omega\left(S U_{\mathbb{K}}(n)\right) \rightarrow \Omega\left(F_{n ; n_{1}, \ldots, n_{k}}^{+}(\mathbb{K})\right) \rightarrow S U_{\mathbb{K}}\left(n_{1}\right) \times S U_{\mathbb{K}}\left(n_{1}-n_{2}\right) \times \cdots \times$ $S U_{\mathbb{K}}\left(n_{k-1}-n_{k}\right) \times S U_{\mathbb{K}}\left(n-n_{k}\right)$, Corollary 1.5, Theorem 1.8 and Proposition 2.2 lead to:

(1) nil $\Omega\left(F_{n ; n_{1}, \ldots, n_{k}}^{+}(\mathbb{R})_{(p)}\right)<\infty$ for $p>2$;

(2) nil $\Omega\left(F_{n ; n_{1}, \ldots, n_{k}}(\mathbb{K})\right)<\infty$ and nil $\Omega\left(F_{n ; n_{1}, \ldots, n_{k}}^{+}(\mathbb{K})\right)<\infty$ for $\mathbb{K}=\mathbb{C}, \mathbb{H}$.

As above, we do not mention above any result on the $p$-localization of $F_{n ; n_{1}, \ldots, n_{k}}(\mathbb{R})$ because we are not sure on its existence.

For the question [4] by Cohen and $\mathrm{Wu}$, we conclude:

Corollary 2.9. For any space $X$ and $m<n \leq \infty$ :

(1) $\operatorname{nil}\left[J(X), \Omega\left(G_{n, m}^{+}(\mathbb{R})_{(p)}\right)\right]<\infty$ for $p \geq 3$;

(2) $\operatorname{nil}\left[J(X), \Omega\left(G_{n, m}(\mathbb{K})\right)\right]<\infty$ for $\mathbb{K}=\bar{C}, \mathbb{H}$;

(3) nil $\left[J(X), \Omega\left(F_{n ; n_{1}, \ldots, n_{k}}^{+}(\mathbb{R})_{(p)}\right)\right]<\infty$ for $p \geq 3$;

(4) nil $\left[J(X), \Omega\left(F_{n ; n_{1}, \ldots, n_{k}}(\mathbb{K})\right)\right]<\infty$ for $\mathbb{K}=\mathbb{C}, \mathbb{H}$ and $1 \leq n_{1}<\cdots<n_{k} \leq$ $n-1$. 
Next, recall the following result of Hopf [7]. Let $X$ be a connected $H$-space with $\operatorname{dim} H^{*}(X ; \mathbb{Q})<\infty$ for the field $\mathbb{Q}$ of rationals. Then, one has a homotopy equivalence

$$
X_{(0)} \simeq \mathbb{S}_{(0)}^{2 n_{1}-1} \times \cdots \times \mathbb{S}_{(0)}^{2 n_{l}-1},
$$

where $-(0)$ means the rationalization.

The sequence $\left(n_{1}, \ldots, n_{l}\right)$ with $n_{1} \leq \cdots \leq n_{l}$ is called the type of $G$. Wilkerson [25] showed that a simply connected compact group $G$ is not $p$-regular for $p<n_{l}$. Then, by combining with Kumpel's result [13] above, $G$ is $p$-regular if and only if $p \geq n_{l}$.

The types of the connected compact simple Lie groups have been summarized in $[18$, Chapter IV] as follows:

\begin{tabular}{|l|l|l|l|}
\hline & & group & type \\
\hline$A_{n}$ & $(n \geq 1)$ & $S U(n+1)$ & $(2,3, \ldots, n+1)$ \\
$B_{n}$ & $(n \geq 2)$ & $S O(2 n+1), \operatorname{Spin}(2 n+1)$ & $(2,4, \ldots, 2 n)$ \\
$C_{n}$ & $(n \geq 3)$ & $S p(n)$ & $(2,4, \ldots, 2 n)$ \\
$D_{n}$ & $(n \geq 4)$ & $S O(2 n), \operatorname{Spin}(2 n)$ & $(3,7, \ldots, 2 n)$ \\
$G_{2}$ & & & $(2,6)$ \\
$F_{4}$ & & & $(2,6,8,12)$ \\
$E_{6}$ & & & $(2,5,6,8,9,12)$ \\
$E_{7}$ & & & $(2,6,8,10,12,14,18)$ \\
$E_{8}$ & & & $(2,8,12,14,18,20,24,30)$ \\
\hline
\end{tabular}

Let $G$ be a compact, connected Lie group of type $\left(n_{1}, \ldots, n_{l}\right)$. By Serre [21], the group $G$ is $p$-regular for an odd prime $p$ if there is a $p$-local (or $p$-complete) homotopy equivalence

$$
G \simeq \mathbb{S}^{2 n_{1}-1} \times \cdots \times \mathbb{S}^{2 n_{l}-1}
$$

Then, by McGibbon [14, Theorem 2], we have:

Theorem 2.11. Let $G$ be a compact connected simple Lie group of type $\left(n_{1}, \ldots, n_{l}\right)$. Then we have:

(1) $G_{(p)}$ is homotopy commutative if $p>2 n_{l}$;

(2) $G_{(p)}$ is not homotopy commutative if $p<2 n_{l}$ except for $(G, p)=$ $(\operatorname{Sp}(2), 3),\left(G_{2}, 5\right)$.

Notice that Table (2.10) and Theorem 2.11 yield:

(1) nil $S O(2 n)_{(p)}=\operatorname{nil} S O(2 n+1)_{(p)}=1$ if $p>4 n$;

(2) $\operatorname{nil} S U(n)_{(p)}=1$ if $p>2 n$;

(3) $\operatorname{nil} \operatorname{Sp}(n)_{(p)}=1 p>4 n$ or $p=3$ and $n=2$.

The McGibbon's result on homotopy commutativity has been generalized by Kaji and Kishimoto [11, Theorem 2] to homotopy nilpotency in the following cases:

Theorem 2.12. Let $G$ be a compact, simply connected, simple Lie group of type $\left(n_{1}, \ldots, n_{l}\right)$ If $G$ is p-regular then $G_{(p)}$ is homotopy nilpotent with: 
(1) $\operatorname{nil} G_{(p)}=2$ if $\frac{3}{2} n_{l}<p<2 n_{l}$;

(2) nil $G_{(p)}=3$ if $n_{l} \leq p \leq \frac{3}{2} n_{l}$ except for the cases that $(G, p)=$ $\left(F_{4}, 17\right),\left(E_{6}, 17\right),\left(E_{8}, 41\right),\left(E_{8}, 43\right)$ or rank $G=1$ with $p=2$;

(3) nil $G_{(p)}=2$ in the above exceptional cases.

Then, Table (2.10) and Theorem 2.12 lead to:

Corollary 2.13. (1) nil $S O(2 n)_{(p)}=\operatorname{nil} S O(2 n+1)_{(p)}=\left\{\begin{array}{l}2 \text { for } 3 n<p<4 n \\ 3 \text { for } 4 n \leq p \leq 3 n\end{array}\right.$

(2) $\operatorname{nil} S U(n)_{(p)}=\left\{\begin{array}{l}2 \text { for } \frac{3}{2} n<p<2 n, \\ 3 \text { for } n \leq p \leq \frac{3}{2} n\end{array}\right.$ when $p$ is odd, and nil $S U(2)_{(2)}=$ $\operatorname{nil} \mathbb{S}_{(2)}^{3}=2$;

(3) $\operatorname{nil} \operatorname{Sp}(n)_{(p)}=\left\{\begin{array}{l}2 \text { for } 3 n<p<4 n \\ 3 \text { for } 2 n \leq p \leq 3 n\end{array}\right.$.

Now, recall that according to Mimura-Toda [17], a compact, connected Lie group $G$ is quasi-p-regular for odd prime $p$ if there is a $p$-local (or $p$-complete) homotopy equivalence $G \simeq \prod_{i=1}^{l} B_{i}$, where each $B_{i}$ is either a sphere or a sphere bundle over a sphere. Recall that by [17, Thorem 4.2], we have:

Theorem 2.14. A Lie group $G$ is quasi-p-regular if and only if:

(1) $p>n$ for $G=\operatorname{Sp}(n)$;

(2) $p>\frac{n}{2}$ for $G=S U(n)$;

(3) $p>\frac{n-1}{2}$ for $G=\operatorname{Spin}(n)$;

(4) $p \geq 5$ for $G=G_{2}, F_{4}, E_{6}$;

(5) $p \geq 11$ for $G=E_{7}, E_{8}$.

Kishimoto [12, Theorem 1.1] determined the homotopy nilpotency class of all quasi- $p$-regular cases of $S U(n)$. The result is:

Theorem 2.15. Let $p>5$ be a prime. Then, we have:

$$
\text { nil } S U(n)_{(p)}=\left\{\begin{array}{l}
3 \text { for } p=n+1 \text { or } \frac{n}{2}<p \leq \frac{2 n+1}{3} \\
2 \text { for } \frac{2 n+1}{3}<p \leq n-2 .
\end{array}\right.
$$

Making use of the above, Lemma 2.1 and the fibration

$$
\Omega\left(U_{\mathbb{K}}(n)_{(p)}\right) \longrightarrow \Omega\left(G_{n, m}(\mathbb{K})_{(p)}\right) \longrightarrow\left(U_{\mathbb{K}}(m)\right)_{(p)} \times\left(U_{\mathbb{K}}(n-m)\right)_{(p)}
$$

for $\mathbb{K}=\mathbb{R}, \mathbb{C}, \mathbb{H}$ and a prime $p$, we may state:

Proposition 2.16. If $1 \leq m \leq n$ then:

(1) $\operatorname{nil} \Omega\left(G_{n, m}(\mathbb{R})_{(p)}\right) \leq 2$ with $p>4 k$ for $n=2 k+1$ or $n=2 k$;

(2) $\quad \operatorname{nil} \Omega\left(G_{n, m}(\mathbb{C})_{(p)}\right) \leq\left\{\begin{array}{l}2 \text { for } p>2 n \\ 3 \text { for } \frac{3}{2} n<p<2(n-m) ; \\ 4 \text { for } n \leq p \leq \frac{3}{2}(n-m) ; \\ 3 \text { for } \frac{2 n+1}{3}<p \leq n-m-2 .\end{array}\right.$

(3) $\operatorname{nil} \Omega\left(G_{n, m}(\mathbb{H})_{(p)}\right) \leq\left\{\begin{array}{l}2 \text { for } p>4 n ; \\ 3 \text { for } 3 n<p<4(n-m) \text {; } \\ 4 \text { for } 2 n \leq p \leq 3(n-m) \text {. }\end{array}\right.$ 


\subsection{Stiefel manifolds}

The Stiefel manifold $V_{n, m}(\mathbb{K})$ is the set of all orthonormal $m$-frames in the vector space $\mathbb{K}^{n}$. That is, it is the set of ordered orthonormal $m$-tuples of vectors in $\mathbb{K}^{n}$ for $\mathbb{K}=\mathbb{R}, \mathbb{C}$ or $\mathbb{H}$.

It is well known that $V_{n, m}(\mathbb{K})$ is a smooth manifold and there are diffeomorphisms:

(1) $V_{n, m}(\mathbb{R})=V_{n, m} \approx O(n) / O(n-m) \approx S O(n) / S O(n-m)$;

(2) $V_{n, m}(\mathbb{C})=W_{n, m} \approx U(n) / U(n-m) \approx S U(n) / S U(n-m)$;

(3) $V_{n, m}(\mathbb{H})=X_{n, m} \approx \operatorname{Sp}(n) / \operatorname{Sp}(n-m)$.

Since the homomorphism $\pi_{1}\left(S U(m)_{\mathbb{K}}\right) \rightarrow \pi_{1}\left(S U(n)_{\mathbb{K}}\right)$ of fundamental groups determined by the inclusion map $S U(m)_{\mathbb{K}} \hookrightarrow S U(n)_{\mathbb{K}}$ for $2 \leq m \leq n$ is an epimorphism, we derive that the spaces $V_{n, m}(\mathbb{K})$ are simply connected for $\mathbb{K}=\mathbb{R}, \mathbb{C}, \mathbb{H}$. Then, Corollary 1.5 and Proposition 2.2 lead to:

Proposition 2.17. If $1 \leq m \leq n$ then:

(1) $\operatorname{nil} \Omega\left(V_{n, m}\right)_{(p)}<\infty$ for $p \geq 3$;

(2) nil $\Omega\left(W_{n, m}\right)<\infty$;

(3) $\operatorname{nil} \Omega\left(X_{n, m}\right)<\infty$.

Then, for the question [4] by Cohen and $\mathrm{Wu}$, we conclude:

Corollary 2.18. If $1 \leq m \leq n$ and $X$ is a space then:

(1) $\operatorname{nil}\left[J(X), \Omega\left(V_{n, m}\right)_{(p)}\right]<\infty$ for $p \geq 3$;

(2) $\operatorname{nil}\left[J(X), \Omega\left(W_{n, m}\right)\right]<\infty$;

(3) $\operatorname{nil}\left[J(X), \Omega\left(X_{n, m}\right)\right]<\infty$.

Making use of Lemma 2.1, Corollary 2.13, Theorem 2.15 and the fibration

$$
\Omega\left(U_{\mathbb{K}}(n)\right)_{(p)} \longrightarrow \Omega\left(V_{n, m}(\mathbb{K})\right)_{(p)} \longrightarrow\left(U_{\mathbb{K}}(n-m)\right)_{(p)}
$$

for $\mathbb{K}=\mathbb{R}, \mathbb{C}, \mathbb{H}$ and a prime $p$, we may state:

Corollary 2.19. If $1 \leq m \leq n$ then:

(1) nil $\Omega\left(V_{n, m}\right)_{(p)} \leq 2$ with $p>k$ for $n-m=2 k+1$ or $n-m=2 k$;

(2) $\operatorname{nil} \Omega\left(W_{n, m}\right)_{(p)} \leq\left\{\begin{array}{l}2 \text { for } p>2(n-m) \\ 3 \text { for } \frac{3}{2}(n-m)<p<2(n-m) \\ 4 \text { for } n-m \leq p \leq \frac{3}{2}(n-m) \\ 4 \text { for } p=n-m+1 \text { or } \frac{n-m}{2}<p \leq \frac{2(n-m)+1}{3} \\ 3 \text { for } \frac{2(n-m)+1}{3}<p \leq n-m-2 .\end{array}\right.$

(3) $\operatorname{nil} \Omega\left(X_{n, m}\right)_{(p)} \leq \begin{cases}2 \text { for } p>4(n-m) \\ 3 & \text { for } 3(n-m)<p<4(n-m) \\ 4 & \text { for } 2(n-m) \leq p \leq 3(n-m)\end{cases}$

Since the spaces $S O(2 n) / U(n), S p(n) / U(n)$ and $S U(2 n) / S p(n)$ are simply connected, Lemma 2.1 and the results above yield: 
Proposition 2.20. If $n \geq 1$ then:

(1) nil $\Omega(S O(2 n) / U(n)))_{(p)}$, nil $\Omega(S p(n) / U(n))_{(p)} \leq\left\{\begin{array}{l}2 \text { for } p>2 n, \\ 3 \text { for } \frac{3}{2} n<p<2 n \text {, } \\ 4 \text { for } n \leq p \leq \frac{3}{2} n\end{array}\right.$

(2) $\operatorname{nil} \Omega(S U(2 n) / S p(n))_{(p)} \leq$

$$
\left\{\begin{array}{l}
2 \text { for } p>4 n \\
3 \text { for } 2 n<p<4 n \text { or } \frac{2 n+1}{3}<p \leq n-2 \text { with } p \geq 5 \\
4 \text { for } 2 n \leq p \leq 3 n, \text { or } p=n+1 \text { or, } \frac{n}{2}<p \leq \frac{2 n+1}{3} \text { with } p \geq 5 .
\end{array}\right.
$$

\section{The Cayley plane $\mathbb{O} P^{2}$ and other homogeneous spaces}

The real Cayley plane $\mathbb{O} P^{2} \approx F_{4} / \operatorname{Spin}(9)$, where $F_{4}$ is a compact form of an exceptional Lie group and $\operatorname{Spin}(9)$ is the spin group of nine-dimensional Euclidean space (realized in $F_{4}$ ).

Since, by means of [19], the group $\operatorname{Spin}(9)$ is not homotopy nilpotent we cannot deduce from the fibration $\Omega\left(F_{4}\right) \longrightarrow \Omega\left(\mathbb{O} P^{2}\right) \longrightarrow$ Spin $(9)$ the homotopy nilpotency of $\mathbb{O} P^{2}$.

Recall that in view of [18], the following is a list of the compact, simply connected, simple groups with $p$-torsion:

\begin{tabular}{|l|l|}
\hline$p$ & \multicolumn{1}{|c|}{ group } \\
\hline 2 & $G_{2}, F_{4}, E_{6}, E_{7}, E_{8}, \operatorname{Spin}(n)$ for $n \geq 7$ \\
3 & $F_{4}, E_{6}, E_{7}, E_{8}$ \\
5 & $E_{8}$ \\
\hline
\end{tabular}

Now, since $\mathbb{O} P^{2}$ is simply connected, Theorem 1.8, Lemma 2.1, Theorem 2.12 and the fibration

$$
\Omega\left(F_{4}\right)_{(p)} \longrightarrow \Omega\left(\mathbb{O} P_{(p)}^{2}\right) \longrightarrow \operatorname{Spin}(9)_{(p)}
$$

for a prime $p$ yield:

Proposition 3.2. (1) If $p \geq 3$ then nil $\mathbb{O} P_{(p)}^{2}<\infty$;

(2) if $p>16$ then nil $\mathbb{O} P_{(p)}^{2} \leq 2$;

(3) nil $\mathbb{O} P_{(p)}^{2} \leq\left\{\begin{array}{l}4 \text { for } p=11 \\ 3 \text { for } p=13 \text {. }\end{array}\right.$

Next, the inclusions

$$
G_{2} \hookrightarrow F_{4} \hookrightarrow E_{6} \hookrightarrow E_{7} \hookrightarrow E_{8}
$$

of exceptional simply connected simple Lie groups lead to fibration:

(1) $\Omega\left(E_{8}\right) \rightarrow \Omega\left(E_{8} / E_{n}\right) \rightarrow E_{n}$ for $n=6,7$;

(2) $\Omega\left(E_{7}\right) \rightarrow \Omega\left(E_{7} / E_{6}\right) \rightarrow E_{6}$;

(3) $\Omega\left(E_{7}\right) \rightarrow \Omega\left(E_{7} / F_{4}\right) \rightarrow F_{4}$;

(4) $\Omega\left(E_{8}\right) \rightarrow \Omega\left(E_{8} / F_{4}\right) \rightarrow F_{4}$; 
(5) $\Omega\left(E_{6}\right) \rightarrow \Omega\left(E_{6} / F_{4}\right) \rightarrow F_{4}$;

(6) $\Omega\left(E_{8}\right) \rightarrow \Omega\left(E_{8} / G_{2}\right) \rightarrow G_{2}$;

(7) $\Omega\left(E_{7}\right) \rightarrow \Omega\left(E_{n} / G_{2}\right) \rightarrow G_{2}$ for $n=6,7$;

(8) $\Omega\left(F_{4}\right) \rightarrow \Omega\left(F_{4} / G_{2}\right) \rightarrow G_{2}$.

Recall that methods developed by Theriault [24, Theorem 12.1] were sufficiently powerful to allow for explicit calculations of the homotopy nilpotency classes of quasi- $p$-regular exceptional Lie groups.

Consequently, the result [24, Theorem 12.1], Theorem 1.8, Lemma 2.1, Theorem 2.12, Table (3.1) and localized fibrations (1)-(8) above lead to:

Proposition 3.3. If $p$ is a prime then:

(1) $\operatorname{nil} \Omega\left(E_{8} / E_{7}\right)_{(p)}< \begin{cases}\infty \text { for } p \geq 5, \\ 3 & \text { for } p>36, \\ 4 & \text { for } p=11,13,17,29,31 \\ 5 & \text { for } p=19,23 ;\end{cases}$

(2) nil $\Omega\left(E_{8} / E_{6}\right)_{(p)}$, nil $\Omega\left(E_{7} / E_{6}\right)_{(p)}<\left\{\begin{array}{l}\infty \text { for } p \geq 5 ; \\ 3 \text { for } p>24, \\ 4 \text { for } p=7,11,19,23, \\ 5 \text { for } p=13,17\end{array}\right.$

(3) nil $\Omega\left(E_{8} / F_{4}\right)_{(p)}, \operatorname{nil} \Omega\left(E_{7} / F_{4}\right)_{(p)}< \begin{cases}\infty \text { for } p \geq 5, \\ 3 & \text { for } p>24, \\ 4 & \text { for } p=7,11,19,23 \text {, } \\ 5 & \text { for } p=13,17 ;\end{cases}$

(4) $\operatorname{nil} \Omega\left(E_{8} / G_{2}\right)_{(p)}, \operatorname{nil} \Omega\left(E_{7} / G_{2}\right)_{(p)}, \operatorname{nil} \Omega\left(E_{6} / G_{2}\right)_{(p)}, \operatorname{nil} \Omega\left(F_{4} / G_{2}\right)_{(p)}<$

$$
\left\{\begin{array}{l}
\infty \text { for } p \geq 3 \\
3 \text { for } p>12 \\
4 \text { for } p=11 \\
5 \text { for } p=7
\end{array}\right.
$$

We close the paper with the following suggested by Stephen Theriault. The homotopy nilpotency class $\Omega(G / K)$ can be sometimes determined precisely when the group $G$ is $p$-regular or quasi- $p$-regular. To aim that we need some prerequisites.

Given an $H$-space $(X, \mu)$, consider the map $t: X \times X \rightarrow X \times X$ given by $t\left(x, x^{\prime}\right)=\left(x^{\prime}, x\right)$ for $x, x^{\prime} \in X$ and write $d_{X}=\mu-\mu t: X \times X \rightarrow X$. Then, $(X, \mu)$ is homotopy commutative if and only if $d_{X}$ is null homotopic.

Lemma 3.4. (1) If an $H$-map $f: X \rightarrow Y$ has a righthomotopyinverse $s: Y \rightarrow X$ and $X$ is homotopy commutative then $Y$ is homotopy commutative as well.

(2) If $F \stackrel{i}{\rightarrow} E \stackrel{q}{\rightarrow} B$ is a fibration and $i: F \rightarrow E$ has a left homotopy inverse then the map $\Omega(q): \Omega(E) \rightarrow \Omega(B)$ has a right homotopy inverse.

Proof.(1): First notice that $d_{Y} \circ(f \times f) \simeq f \circ d_{X}$. Since, $X$ is homotopy commutative we derive that $d_{Y}(f \times f) \simeq *$. Then, the right homotopy inverse $s: Y \rightarrow X$ of $f: X \rightarrow Y$ leads to $d_{Y} \simeq *$.

(2): Since $i: F \rightarrow E$ has a left homotopy, the fibration $F \stackrel{i}{\rightarrow} E \stackrel{q}{\rightarrow} B$ leads to the short exact sequence 


$$
1 \rightarrow[\Omega(B), \Omega(F)] \stackrel{\Omega(i)_{*}}{\longrightarrow}[\Omega(B), \Omega(E)] \stackrel{\Omega(q)_{*}}{\longrightarrow}[\Omega(B), \Omega(B)] \rightarrow 1
$$

which yields a map $s: \Omega(B) \rightarrow \Omega(E)$ such that $\Omega(q) \circ s \simeq \mathrm{id}_{\Omega(B)}$ and the proof is complete.

In particular, if $G$ be a compact Lie group, $K<G$ its closed subgroup and $q: K \rightarrow G / K$ the quotient map then the fibration

$$
\Omega\left(K_{(p)}\right) \stackrel{\Omega(i)}{\rightarrow} \Omega\left(G_{(p)}\right) \stackrel{\Omega(q)}{\longrightarrow} \Omega\left((G / K)_{(p)}\right)
$$

leads to a right homotopy inverse of the loop map $\Omega(q): \Omega\left(G_{(p)}\right) \rightarrow \Omega(G / K)_{(p)}$ provided $G$ is $p$-regular and the canonical map $K_{(p)} \rightarrow G_{(p)}$ has a left homotopy inverse. Since the localized odd sphere $\mathbb{S}_{(p)}^{2 n+1}$ is an associative $H$-space for $p>3$, Lemma 3.4 implies that space $\Omega\left((G / K)_{(p)}\right)$ is homotopy commutative or equivalently nil $\Omega\left((G / K)_{(p)}\right)=1$.

For example, the $p$-local homotopy decompositions of Mimura, Nishida and Toda [16] imply that if $K<G$ is one of:

(1) $S U(m)<S U(n)$ if $m<n$ and $p \geq n$;

(2) $\operatorname{Sp}(m)<\operatorname{Sp}(n)$ if $m<n$ and $p>2 n$;

(3) $\operatorname{Spin}(m)<\operatorname{Spin}(n)$ if $m<n$ and $p>2\left[\frac{n}{2}\right]$;

(4) $G_{2}<F_{4}$ or $G_{2}<E_{6}$ if $p=5$ or $p>11$;

(5) $F_{4}<E_{6}$ if $p>3$;

(6) $F_{4}<E_{7}$ or $G_{2}<E_{7}$ if $p>17$

then the canonical map $K_{(p)} \rightarrow G_{(p)}$ has a left homotopy inverse.

Note that the $p=5$ case in part (4) is quasi- $p$-regular, and the $p=5,7,11$ cases in part (5) are quasi- $p$-regular. The others are all $p$-regular.

Then, we derive:

Corollary 3.5. The following spaces:

(1) $\Omega\left(\left(W_{n, m}\right)_{(p)}\right)=\Omega\left((S U(n) / S U(m))_{(p)}\right)$ if $m<n$ and $p \geq n$;

(2) $\Omega\left(\left(X_{n, m}\right)_{(p)}\right)=\Omega\left((\operatorname{Sp}(n) / S p(m))_{(p)}\right)$ if $m<n$ and $p>2 n$;

(3) $\Omega\left((\operatorname{Spin}(n) / \operatorname{Spin}(m))_{(p)}\right)$ if $m<n$ and $p>2\left[\frac{n}{2}\right]$;

(4) $\Omega\left(\left(F_{4} / G_{2}\right)_{(p)}\right)$ and $\Omega\left(\left(E_{6} / G_{2}\right)_{(p)}\right)$ if $p=5$ or $p>11$;

(5) $\Omega\left(\left(E_{6} / F_{4}\right)_{(p)}\right)$ if $p>3$;

(6) $\Omega\left(\left(E_{7} / F_{4}\right)_{(p)}\right)$ if $p>17$

are homotopy commutative.

Acknowledgements The author is grateful to Stephen Theriault for reading a preliminary version of the manuscript and for allowing to incorporate his valuable comments and suggestions. Even deeper debts are owed to him for suggesting Lemma 3.4 and indicating papers $[11,16,24]$ with the homotopy nilpotency classes of $p$-regular and quasi- $p$-regular exceptional Lie groups, respectively.

The author greatly appreciates the thoughtful comments and suggestions of the anonymous referee. 
Open Access This article is licensed under a Creative Commons Attribution 4.0 International License, which permits use, sharing, adaptation, distribution and reproduction in any medium or format, as long as you give appropriate credit to the original author(s) and the source, provide a link to the Creative Commons licence, and indicate if changes were made. The images or other third party material in this article are included in the article's Creative Commons licence, unless indicated otherwise in a credit line to the material. If material is not included in the article's Creative Commons licence and your intended use is not permitted by statutory regulation or exceeds the permitted use, you will need to obtain permission directly from the copyright holder. To view a copy of this licence, visit http://creativecommons.org/ licenses/by/4.0/.

\section{References}

[1] Arkowitz, M.: Introduction to Homotopy Theory. Universitext. Springer, New York (2011)

[2] Berstein, I., Ganea, T.: Homotopical nilpotency. Illinois J. Math. 5, 99-130 (1961)

[3] Bousfield, A.K., Kan, D.M.: Homotopy Limits, Completions and Localizations. Lecture Notes in Math, vol. 304. Springer, Berlin-New York (1972)

[4] Cohen, F.R., Wu, J.: Private communications, March (2017)

[5] Ganea, T.: On the loop spaces of projective spaces. J. Math. Mech. 16, 853-858 (1967)

[6] Golasiński, M., Gonçalves, D., Wong, P.: Exponents of [ $\left.\Omega\left(\mathbb{S}^{r+1}\right), \Omega(Y)\right]$, pp. 103-122. Trends in Mathematics, Birkhäuser, Algebraic Topology and Related Topics (2019)

[7] Hopf, H.: Uber die Topologie der Gruppen-Mannigfaltigkeiten und ihre Verallgemeinerungen. Ann. Math. 42, 22-52 (1941)

[8] Hopkins, M.: Nilpotence and finite $H$-spaces. Israel J. Math. 66, 238-246 (1989)

[9] Kahn, D.W.: A note on $H$-spaces and Postnikov systems of spheres. Proc. Am. Math. Soc. 15(2), 300-307 (1964)

[10] Kaji, S., Kishimoto, D.: Homotopy nilpotency in p-compact groups (22 Oct, 2007). arXiv:0710.3975v1 [math.AT]

[11] Kaji, S., Kishimoto, D.: Homotopy nilpotency in p-regular loop spaces. Math. Z. 264, 209-224 (2010)

[12] Kishimoto, D.: Homotopy nilpotency in localized $S U(n)$. Homology, Homotopy Appl. 11(1), 61-79 (2009)

[13] Kumpel, P.G.: Mod $p$-equivalences of $\bmod p H$-spaces. Quart. J. Math. 23, 173-178 (1972)

[14] McGibbon, C.: Homotopy commutativity in localized groups. Am. J. Math. 106, 665687 (1984)

[15] Meier, W.: Homotopy nilpotence and localization. Math. Z. 161, 169-183 (1978)

[16] Mimura, M., Nishida, G., Toda, H.: Mod $p$ decomposition of compact lie groups. Publ. Res. Inst. Math. Sci. 13, 627-680 (1977)

[17] Mimura, M., Toda, H.: Cohomology operations and homotopy of compact Lie groups I. Topology 9, 317-336 (1970)

[18] Mimura, M., Toda, H.: “Topology of Lie Groups I, II”. In: Translations of Math. Monographs, 91. AMS Providence, RI (1991)

[19] Rao, V.K.: $\operatorname{Spin}(n)$ is not homotopy nilpotent for $n \geq 7$. Topology 32, 239-249 (1993)

[20] Rao, V.K.: Homotopy nilpotent Lie groups have no torsion in homology. Manuscr. Math. 92, 455-462 (1997)

[21] Serre, J.-P.: Groups d'homotopie et classes groupes d'homotopie. Ann. Math. 58, 258294 (1953) 
[22] Snaith, V.P.: Some nilpotent $H$-spaces. Osaka J. Math. 13, 145-156 (1976)

[23] Stasheff, J.D.: H-spaces from a homotopy point of view. Lecture Notes in Math, vol. 161. Springer, Berlin-Heidelberg-New York (1970)

[24] Theriault, S.: The dual polyhedral product, cocategory and nilpotence. Adv. Math. 340, 138-192 (2018)

[25] Wilkerson, C.W.: K-theory operations and mod $p$ loop spaces. Math. Z. 132, 29-44 (1973)

[26] Yagita, N.: Homotopy nilpotency for simply connected Lie groups. Bull. Lond. Math. Soc. 25, 481-486 (1993)

[27] Zabrodsky, A.: Hopf Spaces. North-Holland Publishing Company, Amsterdam (1976)

Publisher's Note Springer Nature remains neutral with regard to jurisdictional claims in published maps and institutional affiliations. 